\title{
Global Asymptotic Stability of Equilibria in Models for Virus Dynamics
}

\author{
J. Prüss ${ }^{a 1}$, R. Zacher ${ }^{a}$ and R. Schnaubelt ${ }^{b}$ \\ ${ }^{a}$ Institut für Mathematik, Martin-Luther-Universität, D-06099 Halle, Germany \\ ${ }^{b}$ Fakultät für Mathematik, Universität Karlsruhe, D-76128 Karlsruhe, Germany
}

\begin{abstract}
In this paper several models in virus dynamics with and without immune response are discussed concerning asymptotic behaviour. The case of immobile cells but diffusing viruses and T-cells is included. It is shown that, depending on the value of the basic reproductive number $R_{0}$ of the virus, the corresponding equilibrium is globally asymptotically stable. If $R_{0}<1$ then the virus-free equilibrium has this property, and in case $R_{0}>1$ there is a unique disease equilibrium which takes over this property..
\end{abstract}

Key words: May-Nowak model, immune response, diffusion, reproduction number, global asymptotic stability, Lyapunov function

AMS subject classification: 35B40, 92D30

\section{Introduction}

In their recent monograph, May and Nowak [6] considered various models for the dynamics of a virus. They discussed equilibria of their basic model and also in the presence of immune responses of different types, and found the crucial number $R_{0}$, the basic reproductive number of the virus. However, they did not consider the stability and the long-time behaviour of the corresponding dynamical system of 3 or 4 ordinary differential equations.

When working on a model for the dynamics of prions it was discovered in Prüss, Pujo-Menjouet, Webb and Zacher [9] that the basic model of May and Nowak, when scaled appropriately, becomes a special case of the scaled model in [9]. In particular, the Lyapunov functions from [9] are available also for the study of the asymptotic behaviour of the basic model of virus dynamics, see also

\footnotetext{
${ }^{1}$ Corresponding author. E-mail: jan.pruess@mathematik.uni-halle.de
} 
[2], [4]. Thus there arises the natural question whether it is possible to generalize this approach to the case of models taking into account immune response, cf. [6], [11]. In this paper we show that the answer is in the affirmative for two such models.

In the first one T-cells act like predators on the prey -the infected cells- and reproduce like predators in the Volterra-Lotka model. In this case we show that there are two threshold values $R_{0}$ and $R_{i}$. If $R_{0}<1$ then there is no endemic equilibrium, and all solutions converge to the virusfree steady state. If $1<R_{0}<R_{i}$ then there is a unique endemic equilibrium which is globally asymptotically stable, the T-cells die out, and there is no effective immune response. If $R_{0}>R_{i}$ then there is a unique endemic equilibrium with a positive number of T-cells which is globally asymptotically stable; it is here where the immune response is active.

This model has some defects, namely the equilibrium value of the number of infected cells does only depend on the dynamics of the T-cells, not on the dynamics of the virus. Also the immune response becomes active only for large values of $R_{0}$ which either does not seem plausible. For this reason May and Nowak [6] formulated a second model in which the T-cells still act as predators on the infected cells, but do not reproduce as in the Volterra-Lotka model, but are produced in proportion to the number of infected cells. We show in this situation that there is only one threshold value $R_{0}$; for $R_{0}<1$ the virus-free equilibrium is globally asympotically stable and the T-cells die out. For $R_{0}>1$ there is a unique endemic equilibrium which is globally asymptotically stable. In this model the immunse response is always active and leads to the expected behaviour of reducing the number of infected cells.

Both of the above virus models with immune response neglect the spatial movements of the virus and the T-cells, whereas it is reasonable to consider the other cells as immobile. (This matter was not discussed in [6].) Therefore we include in these models the diffusion of the virus and the T-cells, with possibly different diffusivities. In this way we obtain a coupled system of two reaction diffusion equations and of two ordinary differential equations. As always in such problems there arises the interesting question whether the ode-dynamics or the diffusion determine the longterm behavior. We prove that in this case the presence of diffusion does not change the picture given above, in particular there are no spatially heterogeneous equilibria, the threshold numbers retain their values and the spatially homogeneous equilibria keep their stability and attractivity properties, no matter what the diffusivities are.

The plan for this paper is as follows. In sections 2 and 3 we formulate the relevant models and state the main results. The proofs of these results are given in sections 4 and 5. Our arguments rely on Lyapunov functions and, for the pde model, on the theory of semilinear parabolic evolution equations.

\section{Homogeneous models}

May and Nowak introduced the following basic model of virus dynamics for the number $V$ of free viruses, the number $Z$ of noninfected cells and the number $I$ of infected cells, see [6]. Noninfected cells are delivered with a constant rate $\lambda$. Viruses are produced with rate $k I$, and they infect cells with rate $r V Z$. The three species are removed with rates $\nu V, m Z$ and $\mu I$, respectively. These 
hypotheses lead to the May-Nowak system

$$
\begin{aligned}
\dot{V} & =k I-\nu V, \quad t \geq 0, \\
\dot{Z} & =\lambda-m Z-r V Z, \quad t \geq 0, \\
\dot{I} & =r V Z-\mu I, \quad t \geq 0, \\
V(0) & =V_{0}, \quad Z(0)=Z_{0}, \quad I(0)=I_{0},
\end{aligned}
$$

with given constants $\lambda, r, k, m, \mu, \nu>0$ and initial values $V_{0}, Z_{0}, I_{0} \geq 0$. The reproduction rate

$$
R_{0}=\frac{k r \lambda}{m \mu \nu}
$$

is the crucial quantity governing the longterm behavior of the problem (2..1). To describe this fact, we first note that (2..1) has exactly the equilibria

$$
\begin{aligned}
(\bar{V}, \bar{Z}, \bar{I}) & =(0, \lambda / m, 0), \\
\left(V_{*}, Z_{*}, I_{*}\right) & =\left(\frac{k r \lambda-m \mu \nu}{r \mu \nu}, \frac{\mu \nu}{k r}, \frac{\nu}{k} V_{*}\right)=\left(\left(R_{0}-1\right) \frac{m}{r}, \frac{\bar{Z}}{R_{0}},\left(R_{0}-1\right) \frac{m \nu}{r k}\right) .
\end{aligned}
$$

The first one is the disease free equilibrium which is always positive; whereas the second one is strictly positive if and only if $R_{0}>1$. Only in this case $\left(V_{*}, Z_{*}, I_{*}\right)$ is biologically relevant, and then it is called the endemic equilibrium. We further observe that the mean life span of a free virus and an infected cell is $1 / \nu$ and $1 / \mu$, respectively. Moreover, one infected cell added to a population in the disease free equilibrium produces infected cells at a rate of $k r \lambda / m$, approximately. Thus it produces $R_{0}$ newly infected cells in the average. This means that we can expect an outbreak of the infection if and only if $R_{0}>1$. In fact, it can be proved that for $R_{0}<1$ the disease free equilibrium $(\bar{V}, \bar{Z}, \bar{I})$ is stable and attracts each solution of $(2 . .1)$ with positive initial values. If $R_{0}>1$, then $(\bar{V}, \bar{Z}, \bar{I})$ becomes unstable, whereas the endemic equilibrium $\left(V_{*}, Z_{*}, I_{*}\right)$ is stable and attracts each solution starting in $(0,3)^{3}$. (See e.g. [9, Thm.2.3] and the references therein.)

The rather simple model (2..1) describes the early stages of a virus infection quite well, as discussed in [6, §3.4]. However, for $R_{0}>1$ the above indicated result says that $Z(t)$ converges to $Z_{*}=\bar{Z} / R_{0}$ as $t \rightarrow \infty$. For instance, one has $R_{0} \approx 2-5$ for HIV in the framework of the above model, see [6, p.25]. For large $t$ the number of noninfected cells can thus be drastically reduced compared to the disease free equilibrium. This drawback of the basic model (2..1) is easily explained by the fact that it does not take into account the immune response of the infected organism.

An important role in the human immune response is played by cytotoxic T-lymphocytes (or, T-cells) which detect and eliminate infected cells. Their number is denoted by $T$. Following [6, $\S 6]$, we assume that T-cells destroy infected cells with rate sIT and that T-cells are removed with rate $n T$. Concerning the production of T-cells, we first consider the quadratic production rate $a I T$ 
leading to the system

$$
\begin{aligned}
\dot{V} & =k I-\nu V, \quad t \geq 0, \\
\dot{Z} & =\lambda-m Z-r V Z, \quad t \geq 0, \\
\dot{I} & =r V Z-\mu I-s I T, \quad t \geq 0, \\
\dot{T} & =a I T-n T, \quad t \geq 0, \\
V(0) & =V_{0}, \quad Z(0)=Z_{0}, \quad I(0)=I_{0}, \quad T(0)=T_{0},
\end{aligned}
$$

for given constants $\lambda, r, s, a, k, m, n, \mu, \nu>0$ and initial values $V_{0}, Z_{0}, I_{0}, T_{0} \geq 0$. Note that in this model the system $(T, I)$ behaves like a predator-prey system with birth rate of the prey given by the infection rate $r V Z$.

This model reduces to $(2 . .1)$ if $T_{0}=0$. One has the equilibria

$$
\bar{P}=(0, \lambda / m, 0,0) \quad \text { and } \quad P_{*}=\left(V_{*}, Z_{*}, I_{*}, 0\right),
$$

where $V_{*}, Z_{*}, I_{*}$ are given by (2..3) and $P_{*}$ is positive and different from $\bar{P}$ if and only if $R_{0}>1$. There is exactly one more equilibrium, namely

$$
\hat{P}=(\hat{V}, \hat{Z}, \hat{I}, \hat{T})=\left(\frac{k n}{a \nu}, \frac{a \nu \lambda}{a m \nu+r k n}, \frac{n}{a}, \frac{a r k \lambda}{s(a m \nu+r k n)}-\frac{\mu}{s}\right),
$$

which is strictly positive if and only if the second threshold condition

$$
R_{0}>1+\frac{k n r}{a m \nu}=: R_{i}
$$

holds. The next theorem describes the stability and attractivity of the equilibria completely in terms of the numbers $R_{0}$ and $R_{i}$.

Theorem 2..1. Let $\left(V_{0}, Z_{0}, I_{0}, T_{0}\right) \in \mathbb{R}_{+}^{4}$, and let $R_{0}, R_{i}, \bar{P}, P_{*}$ and $\hat{P}$ be given as in (2..2), (2..7), (2..5) and (2..6), respectively. Then the system (2..4) has a unique, positive, bounded solution for all $t \geq 0$.

(a) Let $R_{0}<1$. Then $\bar{P}$ is the unique positive equilibrium of (2..4). It is asymptotically stable and globally attractive in $\mathbb{R}_{+}^{4}$.

(b) Let $1<R_{0}<R_{i}$. Then $\bar{P}$ is unstable in $\mathbb{R}_{+}^{4}$, and $P_{*}$ is the only other positive equilibrium. Further, $P_{*}$ is stable and globally attractive in $(0, \infty)^{4}$.

(c) Let $R_{0}>R_{i}$. Then $\bar{P}$ and $P_{*}$ are unstable in $\mathbb{R}_{+}^{4}$, and $\hat{P}$ is the only other positive equilibrium. Further, $\hat{P}$ is strictly positive, stable, and globally attractive in $(0, \infty)^{4}$.

We observe that for $R_{0}<R_{i}$ the immune response has no influence for large $t$ in so far the solution of (2..4) converges to the equilibrium given by $T=0$ and the equilibrium of the basic virus system (2..1). In this context, notice that the threshold condition $R_{0}<R_{i}$ is equivalent to $I_{*}<n / a$ and that the number of T-cells decreases strictly if and only if $I<n / a$ due to (2..4) 
(where $T>0$, say). Hence, in order to trigger a significant immune response the reproduction rate must be large enough to push $I$ over the critical value $n / a$. In this case it holds

$$
\frac{V_{*}}{\hat{V}}=\frac{I_{*}}{\hat{I}}=\frac{R_{0}-1}{R_{i}-1}>1 \quad \text { and } \quad \frac{Z_{*}}{\hat{Z}}=\frac{R_{i}}{R_{0}}<1 .
$$

Compared with the basic model (2..1), the immune response thus decreases the number of free viruses and of infected cells and increases the number of noninfected cells, roughly by the factor $R_{0} / R_{i}$. Moreover, $\hat{V}$ and $\hat{I}$ are proportional to $1 / a$ and $\hat{T}$ is proportional to $1 / s$; i.e., a more efficient immune system minimizes the impact of the infection using less $\mathrm{T}$-cells. A powerful immune system with $a \gg n$ can compensate the viral attack in the sense that $\hat{Z} \approx \bar{Z}$ in this case.

Nevertheless the above model has two drawbacks. First, it does not describe immune systems reacting also on infections with a small reproduction number. Second, the limit value $\hat{I}=n / a$ does not depend on $s$ nor on the parameters of the virus model. Both shortcomings disappear if one considers a linear production rate $a I$ not depending on the number of T-cells. This appears plausible since the T-cells do not reproduce themselves as in the predator-prey model, but rather are produced and a linear production rate seems reasonable. In this case we have the system

$$
\begin{aligned}
\dot{V} & =k I-\nu V, \quad t \geq 0, \\
\dot{Z} & =\lambda-m Z-r V Z, \quad t \geq 0, \\
\dot{I} & =r V Z-\mu I-s I T, \quad t \geq 0, \\
\dot{T} & =a I-n T, \quad t \geq 0, \\
V(0) & =V_{0}, \quad Z(0)=Z_{0}, \quad I(0)=I_{0}, \quad T(0)=T_{0},
\end{aligned}
$$

for given constants $\lambda, r, s, a, k, m, n, \mu, \nu>0$ and initial values $V_{0}, Z_{0}, I_{0}, T_{0} \geq 0$. Notice that here the number of T-cells also increases initially if $T_{0}=0$ and $V_{0}+I_{0}>0$, independent of the parameters. In this model we have, besides $\bar{P}$, only one more positive equilibrium in the case $R_{0}>1$, namely

$$
\tilde{P}=(\tilde{V}, \tilde{Z}, \tilde{I}, \tilde{T})=\left(\tilde{V}, \frac{\lambda}{m+r \tilde{V}}, \frac{\nu}{k} \tilde{V}, \frac{a \nu}{k n} \tilde{V}\right),
$$

where $\tilde{V}$ is the strictly positive root of the quadratic equation

$$
q(\tilde{V}):=\tilde{V}^{2}+\left(\frac{m}{r}+\frac{k \mu n}{a s \nu}\right) \tilde{V}-\frac{k m n \mu}{a r s \nu}\left(R_{0}-1\right)=0,
$$

which exists due to $R_{0}>1$. Notice that $\tilde{I}$ now depends on all system parameters. Moreover, the following theorem says that the long-term behavior of (2..8) is governed by the threshold $R_{0}=1$ alone.

Theorem 2..2. Let $\left(V_{0}, Z_{0}, I_{0}, T_{0}\right) \in \mathbb{R}_{+}^{4}$, and let $R_{0}, \bar{P}$ and $\tilde{P}$ be given as in (2..2), (2..5) and (2..9), respectively. Then the system (2..8) has a unique, positive, bounded solution for all $t \geq 0$.

(a) Let $R_{0}<1$. Then $\bar{P}$ is the unique positive equilibrium of (2..8). It is stable and globally attractive in $\mathbb{R}_{+}^{4}$.

(b) Let $R_{0}>1$. Then $\bar{P}$ is unstable in $\mathbb{R}_{+}^{4}$ and $\tilde{P}$ is the only other positive equilibrium. Further, $\tilde{P}$ is strictly positive, stable and globally attractive in $(0, \infty)^{4}$. 
Observe that $\tilde{V}<V_{*}$ because $q\left(V_{*}\right)>0$ in (2..10). Therefore the immune response reduces the virus load compared to the basis model (2..1) whenever the infection breaks out. Since (4..4) and (4..5) yield $I_{*} / \tilde{I}=V_{*} / \tilde{V}$, the number of infected cells is reduced by the same ratio. Moreover, the inequality $\tilde{V}<V_{*}=m\left(R_{0}-1\right) / r$ implies that $\tilde{Z}=\lambda(m+r \tilde{V})^{-1}>\lambda\left(m R_{0}\right)^{-1}=Z_{*}$; i.e., the number of noninfected cells is increased by the impact of the T-cells.

\section{Models with diffusion}

Let $G \subset \mathbb{R}^{n}$ be an open bounded domain with $C^{2}$-boundary, $n=n(x)$ denotes the outer normal of $G$ at $x \in \partial G$. In this section we reconsider the models introduced in the previous section, including diffusion of the virus and the T-cells with diffusion constants $d_{V}, d_{T}>0$, while considering the cells as immobile. At the boundary of $G$ we prescribe Neumann boundary conditions, which seem to be the natural ones in this context. In particular, the system (2..4) will be the subsystem resulting from spatially constant initial values, and the equilibria of (2..4) will again be equilibria. For the first model this leads to the following system of reaction diffusion equations.

$$
\begin{aligned}
\partial_{t} V(t, x) & =d_{V} \Delta V(t, x)+k I(t)-\nu V(t, x), \quad t>0, x \in G, \\
\dot{Z}(t) & =\lambda-m Z(t)-r V(t, x) Z(t), \quad t>0, x \in G, \\
\dot{I}(t) & =r V(t, x) Z(t)-\mu I(t)-s I(t) T(t, x), \quad t>0, x \in G, \\
\partial_{t} T(t, x) & =d_{T} \Delta T(t, x)+a I(t) T(t, x)-n T(t, x), \quad t>0, x \in G, \\
\partial_{n} V(t, x) & =\partial_{n} T(t, x)=0, \quad t>0, x \in \partial G, \\
V(0, x) & =V_{0}(x), \quad Z(0)=Z_{0}, \quad I(0)=I_{0}, \quad T(0, x)=T_{0}(x), \quad x \in G,
\end{aligned}
$$

with given constants $\lambda, r, s, a, k, m, n, \mu, \nu>0$, diffusion constants $d_{V}, d_{T}>0$ and initial data $V_{0}, Z_{0}, I_{0}, T_{0} \geq 0$. Note that $T_{0} \equiv 0$ implies $T(t) \equiv 0$ for all $t>0$, hence the basic virus model with diffusing virus is a subsystem of (3..1).

In the second case we similarly have the system

$$
\begin{aligned}
\partial_{t} V(t, x) & =d_{V} \Delta V(t, x)+k I(t)-\nu V(t, x), \quad t>0, x \in G, \\
\dot{Z}(t) & =\lambda-m Z(t)-r V(t, x) Z(t), \quad t>0, x \in G, \\
\dot{I}(t) & =r V(t, x) Z(t)-\mu I(t)-s I(t) T(t, x), \quad t>0, x \in G, \\
\partial_{t} T(t, x) & =d_{T} \Delta T(t, x)+a I(t)-n T(t, x), \quad t>0, x \in G, \\
\partial_{n} V(t, x) & =\partial_{n} T(t, x)=0, \quad t>0, x \in \partial G, \\
V(0, x) & =V_{0}(x), \quad Z(0)=Z_{0}, \quad I(0)=I_{0}, \quad T(0, x)=T_{0}(x), \quad x \in G,
\end{aligned}
$$

with given constants $\lambda, r, s, a, k, m, n, \mu, \nu>0$, diffusion constants $d_{V}, d_{T}>0$ and initial data $V_{0}, Z_{0}, I_{0}, T_{0} \geq 0$.

In the following, we formulate these problems as semilinear evolution equations. As general references for the theory of such problems we recommend the books [3], [5], [7]. The phase space 
is taken as $X=C(\bar{G})^{4}$ and the dependent variable is $P=[V, Z, I, T]^{T} \in X$. Let $D_{N}$ denote the negative Neumann-Laplacian in $C(\bar{G})$, i.e.

$$
D_{N} v=-\Delta v, v \in D\left(D_{N}\right):=\left\{v \in \cap_{p>1} W_{p}^{2}(G): \Delta v \in C(\bar{G}), \partial_{n} v=0 \text { on } \partial G\right\} .
$$

It is well-known that $D_{N}$ is the negative generator of a positive, compact, bounded, and analytic $C_{0}$-semigroup $e^{-D_{N} t}$ in $C(\bar{G})$. The Neumann-Laplacian has the analogous properties in $L_{p}(G)$, $1 \leq p<\infty$. (See e.g. [5, Chap.3], [10] for the corresponding generation results.) Then we define an operator $A$ in $X$ by means of

$$
A=\operatorname{diag}\left(d_{V} D_{N}, 0,0, d_{T} D_{N}\right), \quad D(A)=D\left(D_{N}\right) \times X \times X \times D\left(D_{N}\right) .
$$

$-A$ is the generator of the positive, bounded, and analytic $C_{0}$-semigroup

$$
e^{-A t}=\operatorname{diag}\left(e^{-d_{V} D_{N} t}, I, I, e^{-d_{T} D_{N} t}\right)
$$

which, however, is no longer compact since the diffusivities of $Z$ and $I$ are assumed to be zero. Next we define nonlinear functions $F_{i}$ by means of

$$
\begin{aligned}
& F_{1}(P)=[k I-\nu V, \lambda-m Z-r V Z, r V Z-\mu I-s I T, a I T-n T]^{T}, \\
& F_{2}(P)=[k I-\nu V, \lambda-m Z-r V Z, r V Z-\mu I-s I T, a I-n T]^{T},
\end{aligned}
$$

for $P=[V, Z, I, T]^{T} \in X$. Then we have the abstract reformulation

$$
\dot{P}+A P=F_{i}(P), \quad t>0, P(0)=P_{0},
$$

of (3..1) for $i=1$ and of (3..2) for $i=2$. By the variation of parameters formula from semigroup theory, (3..4) is equivalent to the integral equation

$$
P(t)=e^{-A t} P_{0}+\int_{0}^{t} e^{-A(t-s)} F_{i}(P(s)) d s, \quad t>0 .
$$

Continuous solutions of (3..5) will be called mild solutions of (3..4), as usual. Note that due to the polynomial structure of $F_{i}$ and since we are working in the space of continuous functions, $F_{i}$ is even analytic hence of class $C^{\infty}$. Also note that $F_{i}$ is quasipositive with respect to the standard cone $X_{+}:=C_{+}(\bar{G})^{4}$ in $X$. Therefore the theory of semilinear evolution equations implies that via mild solutions, (3..4) generates a local semiflow in $X$ which is also positivity preserving, $i=1,2$. (See e.g. [3, §3.3].) We show in section 4 that these semiflows are global in $X_{+}$and that their asymptotic behaviour is the same as in the spatially homogeneous case discussed in section 2 . More precisely, we have the following result for (3..1).

Theorem 3..1. Let $\left(V_{0}, Z_{0}, I_{0}, T_{0}\right) \in X_{+}$, and let $R_{0}, R_{i}, \bar{P}, P_{*}$ and $\hat{P}$ be given as in (2..2), (2..7), (2..5) and (2..6), respectively. Then the system (3..1) has a unique, positive, bounded mild solution $P \in C\left(\mathbb{R}_{+} ; X\right)$.

(a) Let $R_{0}<1$. Then $\bar{P}$ is the unique positive equilibrium of (3..1). It is asymptotically stable and 
globally attractive in $X_{+}$.

(b) Let $1<R_{0}<R_{i}$. Then $\bar{P}$ is unstable in $X_{+}$, and $P_{*}$ is the only other positive equilibrium. Further, $P_{*}$ is stable and globally attractive in $X_{+} \backslash\left(\{0\} \times C_{+}(\bar{G}) \times\{0\}^{2}\right)$.

(c) Let $R_{0}>R_{i}$. Then $\bar{P}$ and $P_{*}$ are unstable in $X_{+}$, and $\hat{P}$ is the only other positive equilibrium. Further, $\hat{P}$ is strictly positive, stable, and globally attractive in $X_{+} \backslash\left(C_{+}(\bar{G})^{3} \times\{0\}\right)$.

(d) Let $R_{0}>1$. Then for the subsystem $T \equiv 0$, the equilibrium $\bar{P}$ is unstable, and $P_{*}$ is stable and globally attractive in $C_{+}(\bar{G})^{3} \backslash\left(\{0\} \times C_{+}(\bar{G}) \times\{0\}\right)$.

For the second model (3..2) our main result reads as follows.

Theorem 3..2. Let $\left(V_{0}, Z_{0}, I_{0}, T_{0}\right) \in X_{+}$, and let $R_{0}, \bar{P}$ and $\tilde{P}$ be given as in (2..2), (2..5) and (2..9), resepctively. Then the system (3..2) has a unique, positive, bounded mild solution $P \in$ $C\left(\mathbb{R}_{+} ; X\right)$.

(a) Let $R_{0}<1$. Then $\bar{P}$ is the unique positive equilibrium of (3..2). It is stable and globally attractive in $X_{+}$.

(b) Let $R_{0}>1$. Then $\bar{P}$ is unstable in $X_{+}$and $\tilde{P}$ is the only other positive equilibrium. Further, $\tilde{P}$ is strictly positive, stable and globally attractive in $X_{+} \backslash\left(\{0\} \times C_{+}(\bar{G}) \times\{0\} \times C_{+}(\bar{G})\right)$.

We would like to point out that these results in particular imply that in both cases there are no spatially heterogeneous equilibria.

\section{Proofs of Theorem 2.1 and 2.2}

Before proving Theorem $2 . .1$ we rescale the system (2..4) by setting

$$
\begin{aligned}
x(t) & =\frac{r}{\mu} V(t / \mu), \quad y(t)=\frac{k r}{\mu^{2}} Z(t / \mu), \quad z(t)=\frac{k r}{\mu^{2}} I(t / \mu), \quad w(t)=\frac{s}{\mu} T(t / \mu), \\
x_{0} & =\frac{r V_{0}}{\mu}, \quad y_{0}=\frac{k r Z_{0}}{\mu^{2}}, \quad z_{0}=\frac{k r I_{0}}{\mu^{2}}, \quad w_{0}=\frac{s T_{0}}{\mu}, \\
\xi & =\frac{\nu}{\mu}, \quad \sigma=\frac{k r \lambda}{\mu^{3}}, \quad \rho=\frac{m}{\mu}, \quad \alpha=\frac{a \mu}{k r}, \quad \beta=\frac{n}{\mu} .
\end{aligned}
$$

Then (2..4) is equivalent to the problem

$$
\begin{aligned}
\dot{x} & =z-\xi x, \quad t \geq 0, \\
\dot{y} & =\sigma-\rho y-x y, \quad t \geq 0, \\
\dot{z} & =x y-z-w z, \quad t \geq 0, \\
\dot{w} & =\alpha w z-\beta w, \quad t \geq 0, \\
x(0) & =x_{0}, \quad y(0)=y_{0}, \quad z(0)=z_{0}, \quad w(0)=w_{0},
\end{aligned}
$$

with given initial values $x_{0}, y_{0}, z_{0}, w_{0} \geq 0$ and constants $\alpha, \beta, \xi, \sigma, \rho>0$. The threshold condition $R_{0}>1$ is equivalent to $\sigma>\rho \xi$, and $R_{0}>R_{i}$ is equivalent to $\sigma-\rho \xi>\beta / \alpha$. In the new variables 
we have the equilibria

$$
\begin{aligned}
\bar{p} & =(\bar{x}, \bar{y}, \bar{z}, \bar{w})=\left(0, \frac{\sigma}{\rho}, 0,0\right), \\
p_{*} & =\left(x_{*}, y_{*}, z_{*}, w_{*}\right)=\left(\frac{\sigma}{\xi}-\rho, \xi, \sigma-\rho \xi, 0\right), \\
\hat{p} & =(\hat{x}, \hat{y}, \hat{z}, \hat{w})=\left(\frac{\beta}{\alpha \xi}, \frac{\sigma}{\rho+\hat{x}}, \frac{\beta}{\alpha}, \frac{\hat{y}}{\xi}-1\right),
\end{aligned}
$$

where $p_{*}$ is positive if $\sigma>\rho \xi$ and $\hat{p}$ is positive if $\sigma-\rho \xi>\beta / \alpha$.

Proof of Theorem 2..1. It suffices to consider the normalized system (4..2) with strictly positve constants and positive initial values. It is clear that this problem possesses a unique positive solution $(x, y, z, w)$. We set $u=x / 2+y+z+w / \alpha$ und $\kappa=\min \{\xi, \rho, 1 / 2, \beta\}>0$. Then (4..2) yields the inequality

$$
\dot{u}=\sigma-\xi x / 2-\rho y-z / 2-\beta w / \alpha \leq \sigma-\kappa u,
$$

so that $u \leq u(0)+\sigma / \kappa$ by integration. Therefore the solutions are bounded and exist for all $t \geq 0$. We recall that the equilibria of (4..2) are given by (4..3), (4..4) and (4..5). Denoting the right hand side of (4..2) by $f(x, y, z, w)$, we have

$$
\begin{aligned}
f^{\prime}(\bar{p}) & =\left(\begin{array}{cccc}
-\xi & 0 & 1 & 0 \\
-\sigma / \rho & -\rho & 0 & 0 \\
\sigma / \rho & 0 & -1 & 0 \\
0 & 0 & 0 & -\beta
\end{array}\right), \\
f^{\prime}\left(p_{*}\right) & =\left(\begin{array}{cccc}
-\xi & 0 & 1 & 0 \\
-\xi & -\rho-x_{*} & 0 & 0 \\
\xi & x_{*} & -1 & -z_{*} \\
0 & 0 & 0 & \alpha z_{*}-\beta
\end{array}\right) .
\end{aligned}
$$

In Subsection 3.2 of [9] the left upper $3 \times 3$ sub-matrices $A$ of $f^{\prime}(\bar{p})$ and $B$ of $f^{\prime}\left(p_{*}\right)$ were considered. There it was shown that the eigenvalues of $A$ have strictly negative real parts if $\sigma<\rho \xi$ and that the eigenvalues of $B$ have strictly negative real parts if $\sigma>\rho \xi$. In view of (4..6) and the principle of linearized stability, it follows that $\bar{p}$ is stable if $\sigma<\rho \xi$ and that $p_{*}$ is stable if $0<z_{*}=\sigma-\rho \xi<\beta / \alpha$, cp. (4..4). The instability assertions for these two equilibria will be a direct consequence of the attractivity properties of $p_{*}$ and $\hat{p}$ shown below.

First, let $\sigma<\rho \xi$. To treat $\bar{p}$, we introduce the function

$$
\Phi_{0}(x, y, z, w)=\frac{1}{2}(y-\bar{y})^{2}+(2 \xi-\bar{y})\left(x+z+\frac{1}{\alpha} w\right) .
$$

Then (4..2) and $\sigma=\rho \bar{y}$ easily yield

$$
\dot{\Phi}_{0}(x, y, z, w)=-\rho(y-\bar{y})^{2}-x\left[(y-\xi)^{2}+\xi(\xi-\bar{y})\right]-\beta(2 \xi-\bar{y}) w / \alpha .
$$

Since $x, w \geq 0$ and $\bar{y}=\sigma / \rho<\xi$, we obtain that $\Phi_{0}$ is a Lyapunov function. If $\dot{\Phi}_{0}(x, y, z, w)=0$, then it follows $y=\bar{y}, x=0$ and $w=0$, so that $z=0$ by the first line of (4..2). As a result, $\Phi_{0}$ is a strict Lyapunov function on $\mathbb{R}_{+}^{4}$ and so $\bar{p}$ attracts all solutions starting in $\mathbb{R}_{+}^{4}$. 
Second, let $z_{*}=\sigma-\rho \xi \in(0, \beta / \alpha)$, and consider the function

$$
\Phi_{*}(x, y, z, w)=x-x_{*} \ln x+y-y_{*} \ln y+z-z_{*} \ln z+w / \alpha .
$$

Here we assume that $x, y, z>0$. We deduce from (4..2) and the computations in $[9, \S 3.4]$ that

$$
\dot{\Phi}_{*}(x, y, z, w)=-\frac{\rho}{y}\left(y-y_{*}\right)^{2}-x_{*}\left[\frac{z}{x}+\frac{\xi^{2}}{y}+\frac{\xi x y}{z}-3 \xi\right]-w z+w z_{*}+w z-\frac{\beta w}{\alpha} .
$$

As in $[9, \S 3.4]$ we set $a=z / x>0, b=\xi^{2} / y>0$, and $\varphi(u, v)=u+v+\frac{\xi^{3}}{u v}-3 \xi$ for $u, v>0$. Then the term in brackets $[\cdots]$ is equal to $\varphi(a, b)$. On the other hand, it can be seen that $\varphi(\xi, \xi)=0$ is the unique strict minimum of $\varphi$ on $(0, \infty)^{2}$. Since $z_{*}<\beta / \alpha$, we arrive at

$$
\dot{\Phi}_{*}(x, y, z, w) \leq-\frac{\rho}{y}\left(y-y_{*}\right)^{2}-\left(\frac{\beta}{\alpha}-z_{*}\right) w \leq 0 .
$$

So $\Phi_{*}$ is a Lyapunov function for $(4 . .2)$ on $(0, \infty)^{4}$. If $\dot{\Phi}_{*}(x, y, z, w)=0$, then $y=y_{*}$ and $w=0$. So the differential equations (4..2) yield $x=x_{*}$ and $z=z_{*}$, and $\Phi_{*}$ is a strict Lyapunov function. Let $x_{0}, y_{0}, z_{0}>0, w_{0} \geq 0$, and $c=\max \left\{\Phi_{*}\left(x_{0}, y_{0}, z_{0}, w_{0}\right), \Phi_{*}\left(p_{*}\right)\right\}$. The level set $N=$ $\left\{(x, y, z, w) \in(0, \infty)^{3} \times \mathbb{R}_{+}: \Phi_{*}(x, y, z, w) \leq c\right\}$ is closed and invariant since $\Phi_{*}(x, y, z, w) \rightarrow$ $\infty$ as one of the components $x, y, z$ approaches 0 . Moreover, $N$ contains exactly the equilibrium $p_{*}$. Therefore the solution converges to $p_{*}$ as $t \rightarrow \infty$ thanks to La Salle's principle.

Third, let $\sigma-\rho \xi>\beta / \alpha$ and define

$$
\Phi(x, y, z, w)=\frac{\hat{y}}{\xi}(x-\hat{x} \ln x)+(y-\hat{y} \ln y)+(z-\hat{z} \ln z)+\frac{1}{\alpha}(w-\hat{w} \ln w)
$$

for $x, y, z, w>0$. Due to (4...5), it holds $\hat{z}=\xi \hat{x}=\beta / \alpha$ and $1+\hat{w}=\hat{y} / \xi$. Using these relations and (4..2), we calculate

$$
\begin{aligned}
\dot{\Phi}(x, y, z, w)= & \frac{\hat{y} z}{\xi}-\hat{y} x-\frac{\hat{x} \hat{y} z}{\xi x}+\hat{x} \hat{y}-\frac{\rho}{y}(y-\hat{y})^{2}+\hat{x} \hat{y}-x y-\frac{\hat{x} \hat{y}^{2}}{y}+\hat{y} x \\
& +x y-z-w z-\frac{\xi \hat{x} x y}{z}+\xi \hat{x}+\frac{\beta w}{\alpha}+w z-\frac{\beta w}{\alpha}-\hat{w} z+\frac{\beta \hat{w}}{\alpha} \\
= & -\frac{\rho}{y}(y-\hat{y})^{2}-\frac{\hat{x} \hat{y} z}{\xi x}+2 \hat{x} \hat{y}-\frac{\hat{x} \hat{y}^{2}}{y}-\frac{\xi \hat{x} x y}{z}+\xi \hat{x}+\frac{\beta \hat{w}}{\alpha} \\
= & -\frac{\rho}{y}(y-\hat{y})^{2}-\hat{x}\left[\frac{\hat{y} z}{\xi x}+\frac{\hat{y}^{2}}{y}+\frac{\xi x y}{z}-2 \hat{y}-\xi\right]+\frac{\beta \hat{w}}{\alpha} .
\end{aligned}
$$

We set $a=z / x>0, b=\xi \hat{y} / y>0$, and $\psi(u, v)=u+v+\frac{\xi^{3}}{u v}-2 \xi-\xi^{2} / \hat{y}$ for $u, v>0$. Then we obtain $[\cdots]=\psi(a, b) \hat{y} / \xi$ and $\psi=\varphi+\xi(1-\xi / \hat{y})$ for the positive function $\varphi$ defined above. Hence,

$$
\begin{aligned}
\dot{\Phi}(x, y, z, w) & \leq-\frac{\rho}{y}(y-\hat{y})^{2}-\frac{\hat{x} \hat{y}}{\xi} \frac{\xi}{\hat{y}}(\hat{y}-\xi)+\frac{\beta \hat{w}}{\alpha}=-\frac{\rho}{y}(y-\hat{y})^{2}-\xi \hat{x} \hat{w}+\frac{\beta \hat{w}}{\alpha} \\
& =-\frac{\rho}{y}(y-\hat{y})^{2} \leq 0
\end{aligned}
$$


and $\Phi$ is a Lyapunov function on $(0, \infty)^{4}$. Observe that $\hat{p}$ is a strict minimum of $\Phi$ in $(0, \infty)^{4}$. This fact shows the stability of $\hat{p}$. Assume that $\dot{\Phi}(x, y, z, w)=0$. Then $y=\hat{y}$, and the second equation in (4..2) yields that $x$ is constant. Hence, $z$ and $w$ are also constant due to the first and third equation in (4..2). Therefore $\Phi$ is a strict Lyapunov function. Let $x_{0}, y_{0}, z_{0}, w_{0}>0$ and $c=\max \left\{\Phi\left(x_{0}, y_{0}, z_{0}, w_{0}\right), \Phi(\hat{p})\right\}$. As above we see that $N=\left\{(x, y, z, w) \in(0, \infty)^{4}\right.$ : $\Phi(x, y, z, w) \leq c\}$ is closed, invariant, and contains exactly the equilibrium $\hat{p}$. By La Salle's principle, the solution converges to $p_{*}$ as $t \rightarrow \infty$.

We scale the system (2..8) as in (4..1), but now using the constant $\alpha^{\prime}=a s /(k r)$. This leads to the normalized problem

$$
\begin{aligned}
\dot{x} & =z-\xi x, \quad t \geq 0, \\
\dot{y} & =\sigma-\rho y-x y, \quad t \geq 0, \\
\dot{z} & =x y-z-w z, \quad t \geq 0, \\
\dot{w} & =\alpha^{\prime} z-\beta w, \quad t \geq 0, \\
x(0) & =x_{0}, \quad y(0)=y_{0}, \quad z(0)=z_{0}, \quad w(0)=w_{0},
\end{aligned}
$$

with given initial values $x_{0}, y_{0}, z_{0}, w_{0} \geq 0$ and constants $\alpha^{\prime}, \beta, \xi, \sigma, \rho>0$. In this case we have again the equilibrium $\bar{p}$ from (4..3). If $\sigma>\rho \xi$, there also exists the positive equilibrium

$$
\tilde{p}=(\tilde{x}, \tilde{y}, \tilde{z}, \tilde{w})=\left(\tilde{x}, \frac{\sigma}{\rho+\tilde{x}}, \xi \tilde{x}, \frac{\alpha^{\prime} \xi \tilde{x}}{\beta}\right)
$$

where $\tilde{x}$ is the unique positive root of the quadratic equation

$$
\tilde{x}^{2}+\left(\rho+\frac{\beta}{\alpha^{\prime} \xi}\right) \tilde{x}+\frac{\beta}{\alpha^{\prime} \xi^{2}}(\rho \xi-\sigma)=0 .
$$

Proof of Theorem 2..2. Again, we only have to consider the rescaled system (4..7) with strictly positive constants and positive initial values. Clearly, there is a unique positive solution $(x, y, z, w)$. We set $u=x / 3+y+z+w /\left(3 \alpha^{\prime}\right)$ and $\kappa=\min \{\xi, \rho, 1 / 3, \beta\}>0$. Then (4..7) yields the estimate

$$
\dot{u}=\sigma-\frac{\xi}{3} x-\rho y-\frac{1}{3} z-w z-\frac{\beta}{3 \alpha^{\prime}} w \leq \sigma-\kappa u,
$$

so that $u \leq u(0)+\sigma / \kappa$ by integration. As result, the solution is bounded and exists for all times. The (in-)stability of the equilibrium $\bar{p}$ can be shown as in Theorem 2..1. Let $\sigma<\rho \xi$. Using now the function

$$
\Phi_{0}(x, y, z, w)=\frac{1}{2}(y-\bar{y})^{2}+(2 \xi-\bar{y})\left(x+z+\frac{1}{2 \alpha^{\prime}} w^{2}\right),
$$

we prove the global attractivity of $\bar{p}$ as in Theorem $2 . .1$.

Let $\sigma>\rho \xi$ and consider the equilibrium $\tilde{p}$ from (4..8). To establish its attractivity, we introduce the function

$$
\Phi(x, y, z, w)=\frac{\tilde{y}}{\xi}(x-\tilde{x} \ln x)+(y-\tilde{y} \ln y)+(z-\tilde{z} \ln z)+\frac{1}{2 \alpha^{\prime}}(w-\tilde{w})^{2}
$$


for $x, y, z>0$ and $w \geq 0$. It holds $\tilde{z}=\xi \tilde{x}=\beta \tilde{w} / \alpha^{\prime}$ and $1+\tilde{w}=\tilde{y} / \xi$ due to (4..8) and the third equation in (4..7). Taking into account these relations and (4..7), we compute

$$
\begin{aligned}
\dot{\Phi}(x, y, z, w)= & \frac{\tilde{y} z}{\xi}-\tilde{y} x-\frac{\tilde{x} \tilde{y} z}{\xi x}+\tilde{x} \tilde{y}-\frac{\rho}{y}(y-\tilde{y})^{2}+\tilde{x} \tilde{y}-x y-\frac{\tilde{x} \tilde{y}^{2}}{y}+\tilde{y} x \\
& \quad+x y-z-w z-\frac{\xi \tilde{x} x y}{z}+\xi \tilde{x}+\xi \tilde{x} w+w z-\tilde{w} z-\frac{\beta w}{\alpha^{\prime}}(w-\tilde{w}) \\
= & -\frac{\rho}{y}(y-\tilde{y})^{2}-\frac{\tilde{x} \tilde{y} z}{\xi x}+2 \tilde{x} \tilde{y}-\frac{\tilde{x} \tilde{y}^{2}}{y}-\frac{\xi \tilde{x} x y}{z}+\xi \tilde{x}(1+\tilde{w})-\frac{\beta}{\alpha^{\prime}}(w-\tilde{w})^{2} \\
= & -\frac{\rho}{y}(y-\tilde{y})^{2}-\frac{\beta}{\alpha^{\prime}}(w-\tilde{w})^{2}-\frac{\tilde{x} \tilde{y}}{\xi}\left[\frac{z}{x}+\frac{\xi \tilde{y}}{y}+\frac{\xi^{2} x y}{\tilde{y} z}-3 \xi\right] .
\end{aligned}
$$

Setting $a=z / x>0$ and $b=\xi \tilde{y} / y>0$, we obtain $[\cdots]=a+b+\frac{\xi^{3}}{a b}-3 \xi=\varphi(a, b)$, where $\varphi$ is positive by the proof of Theorem 2..1. So we have shown that

$$
\dot{\Phi}(x, y, z, w) \leq-\frac{\rho}{y}(y-\tilde{y})^{2}-\frac{\beta}{\alpha^{\prime}}(w-\tilde{w})^{2},
$$

and that $\Phi$ is a Lyapunov function on $(0, \infty)^{3} \times \mathbb{R}_{+}$. Since $\tilde{p}>0$ is a strict local minimum of $\Phi$, we derive that $\tilde{p}$ is stable. Let $\dot{\Phi}(x, y, z, w)=0$. It follows that $y=\tilde{y}$ and $w=\tilde{w}$. Because of (4..7), also $x$ and $z$ are constant. Thus $\Phi$ is a strict Lyapunov function. As in the proof of Theorem 2..1, we can now establish the asserted global attractivity of $\tilde{p}$.

\section{Proofs of Theorem 3.1 and 3.2}

Proof. We begin with the proof of Theorem 3.2. We perform the scaling as in Section 4 and obtain for $p=[u, y, z, w]^{T} \in X$ the problem

$$
\dot{p}+A p=F_{2}(p), \quad t>0, p(0)=p_{0},
$$

where $F_{2}$ denotes the right hand side of (4..7), with $x$ replaced by $u$, and here

$$
A=\operatorname{diag}\left(\delta_{u} D_{N}, 0,0, \delta_{w} D_{N}\right), \quad D(A)=D\left(D_{N}\right) \times X \times X \times D\left(D_{N}\right),
$$

with $\delta_{i}=d_{i} / \mu$. As mentioned above, there is a unique mild solution of (5..1) which exists on a maximal time interval $\left[0, t_{\max }\right)$, and it is in $X_{+}$if the initial value is so.

(i) Regularity

Since $e^{-A t}$ is an analytic $C_{0}$-semigroup and $F_{2}$ is of class $C^{1}$ we obtain $p \in C^{\gamma}\left(\left(0, t_{\max }\right) ; X\right) \cap$ $C\left(\left(0, t_{\max }\right) ; D\left(A^{\gamma}\right)\right)$, for each $\gamma \in(0,1)$. The equations for $y$ and $z$ then imply immediately $y, z \in C^{1}\left(\left[0, t_{\max }\right) ; C(\bar{G})\right)$. On the other hand, parabolic regularity theory yields further $u, w \in$ $C^{1}\left(\left(0, t_{\max }\right) ; C(\bar{G})\right)$ and $\Delta u, \Delta w \in C\left(\left(0, t_{\max }\right) ; C(\bar{G})\right)$, see e.g. [5, Thm.4.3.4]. Thus for $t>0$ we have classical solutions. 
(ii) Uniform Bounds

To obtain global existence we have to prove boundedness of $p(t)$ on its interval of existence. For this we proceed as follows. Assume $p_{0} \in X_{+}$. Then adding the equations for $y$ and $z$ we obtain

$$
\partial_{t}(y+z)=\sigma-\rho y-z-w z \leq \sigma-\rho_{0}(y+z),
$$

where $\rho_{0}=\min \{\rho, 1\}$ since $p \in X_{+}$. This implies

$$
0 \leq(y+z)(t) \leq e^{-\rho_{0} t}\left(y_{0}+z_{0}\right)+\left(1-e^{-\rho_{0} t}\right) \sigma / \rho_{0},
$$

which shows that $y$ and $z$ are uniformly bounded. Next we look at the equation for $u$, it reads

$$
\dot{u}+\delta_{u} D_{N} u+\xi u=z, \quad u(0)=u_{0} .
$$

Obviously, boundedness of $e^{-\delta_{u} D_{N} t}$ and of $z$ in $C(\bar{G})$ and $\xi>0$ yield uniform boundedness of $u$ as well. The same argument applies also to $w$, here we use $\beta>0$. Hence we may conclude that $p(t)$ is bounded in $X$, with bound depending only on the parameters and the $L_{\infty}$-norms of the initial values. This implies global existence and boundedness of $p(t)$ on $\mathbb{R}_{+}$in $X_{+}$, due to e.g. [5, Prop.7.1.8].

(iii) Compactness

Since $e^{-\delta_{i} D_{N} t}$ is bounded and analytic, the uniform boundedness of $z(t)$ in $X$ as well as $\xi, \beta>0$ imply that $u(t)$ and $w(t)$ are uniformly bounded in $D\left(D_{N}^{1 / 2}\right)$ for $t \geq 1$. Therefore, the orbits $\gamma(u):=u\left(\mathbb{R}_{+}\right) \subset X_{+}$and $\gamma(w):=w\left(\mathbb{R}_{+}\right) \subset X_{+}$are relatively compact, and so the limit sets

$$
\omega(v)=\left\{v_{\infty} \in X: v\left(t_{n}\right) \rightarrow v_{\infty} \text { for some sequence } t_{n} \rightarrow \infty\right\}
$$

for $v=u$ and $v=w$ are nonempty, compact and connected, see [3, $\S 4.3]$. By the theorem of Arzela-Ascoli this implies that the sets $\gamma(u)$ and $\gamma(w)$ are equi-continuous on $\bar{G}$. We want to show that the orbits of $y$ and $z$ are relatively compact, as well. Since we already have boundedness in $C(\bar{G})$ we need to show equi-continuity, employing again the Arzela-Ascoli theorem. For this purpose we subtract the equations for $y$ at two different points $x$ and $\bar{x}$ to the result

$$
\partial_{t}[y(t, x)-y(t, \bar{x})]+(\rho+u(t, x))[y(t, x)-y(t, \bar{x})]=-y(t, \bar{x})[u(t, x)-u(t, \bar{x})] .
$$

Since $u(t, x) \geq 0$ and $0 \leq y(t, \bar{x}) \leq C<\infty$, this implies

$$
|y(t, x)-y(t, \bar{x})| \leq e^{-\rho t}\left|y_{0}(x)-y_{0}(\bar{x})\right|+C \int_{0}^{t} e^{-\rho(t-s)}|u(s, x)-u(s, \bar{x})| d s,
$$

which easily implies equi-continuity of $\gamma(y)$. Similarly, we have

$$
\begin{aligned}
\partial_{t}[z(t, x)-z(t, \bar{x})] & +(1+w(t, x))[z(t, x)-z(t, \bar{x})] \\
= & -z(t, \bar{x})[w(t, x)-w(t, \bar{x})]+[(u y)(t, x)-(u y)(t, \bar{x})],
\end{aligned}
$$


which also yields equi-continuity of $\gamma(z)$ since $w(t, x) \geq 0, p$ is bounded and $\gamma(u), \gamma(y)$ and $\gamma(w)$ are already known to be equi-continuous. Therefore $\omega(y)$ and $\omega(z)$ are also nonempty, compact and connected, hence $\omega(p) \subset X_{+}$has these properties as well.

(iv) Strong Positivity

Another important property of the semiflow is its strong positivity. Since $\sigma>0$, we have $y(t, x)>$ 0 for all $t>0, x \in \bar{G}$, even if $y_{0} \equiv 0$; in fact we have the explicit lower bound

$$
y(t, x) \geq e^{-(\rho+C) t} y_{0}(x)+\left(1-e^{-(\rho+C) t}\right) \sigma /(\rho+C), \quad t>0, x \in \bar{G},
$$

where $C=\sup _{t>0, x \in G} u(t, x)$. Next we know $u(t, x) \geq 0$ for all $t \geq 0$ and $x \in \bar{G}$. Then the strong maximum principle and Hopf's lemma (cf. [8, §3.3]) imply that in case $u\left(t_{0}, x_{0}\right)=0$ for some $t_{0}>0, x_{0} \in \bar{G}$ we have $u_{0} \equiv 0$ as well as $z \equiv 0$ on $\left(0, t_{0}\right) \times \bar{G}$ which implies $z_{0} \equiv 0$ by continuity. Thus $u(t, x)>0$ for all $t>0$ and $x \in \bar{G}$ unless $u_{0} \equiv z_{0} \equiv 0$. If the latter is not the case then $(u y)(t, x)>0$, hence also $z(t, x)>0$ for all $t>0, x \in \bar{G}$, which finally yields $w(t, x)>0$ for all such $t$ and $x$. This shows strong positivity of the solutions. As a consequence, there are two possibilities: either $u_{0} \equiv z_{0} \equiv 0$, then $u(t) \equiv z(t) \equiv 0$ for all $t>0$ and $y(t) \rightarrow \bar{y}:=\sigma / \rho$ and $w(t) \rightarrow 0$ in $C(\bar{G})$ exponentially as $t \rightarrow \infty$, or $u_{0}+z_{0} \neq 0$ and this implies $p(t) \in \operatorname{int} X_{+}$for all $t>0$. In particular this shows that int $X_{+}$is a positive invariant set for the semiflow, and that we may restrict attention to initial values in int $X_{+}$.

(v) The Case $R_{0}<1$

Suppose $R_{0}<1$. We define a functional $\Phi_{0}(p)$ by means of

$$
\Phi_{0}(p)=\int_{G}\left[\frac{1}{2}(y-\bar{y})^{2}+(2 \xi-\bar{y})\left(u+z+\frac{1}{2 \alpha^{\prime}} w^{2}\right] d x .\right.
$$

This functional is well-defined and of class $C^{1}$ on $X$. A simple computation yields the derivative of $\Phi_{0}$ along a solution.

$$
\begin{aligned}
\partial_{t} \Phi_{0}(p(t))= & -\frac{\delta_{w}}{\alpha^{\prime}}(2 \xi-\bar{y}) \int_{G}|\nabla w|^{2} d x-\frac{\beta}{\alpha^{\prime}} \int_{G} w^{2} d x \\
& -\rho \int_{G}(y-\bar{y})^{2} d x-\int_{G} u\left[(y-\xi)^{2}+\xi^{2}\left(1-R_{0}\right)\right] d x \leq 0,
\end{aligned}
$$

since $R_{0}=\sigma / \xi \rho<1$ by assumption. Thus $\Phi_{0}$ is a Lyapunov functional for (5..1), and it is even a strict Lyapunov functional since $\partial_{t} \Phi_{0}(p)=0$ implies $w \equiv 0, y \equiv \bar{y}$ and $u \equiv 0$ hence also $z \equiv 0$ by the equations. Therefore the limit set $\omega(p)$ equals $\{\bar{p}\}$, i.e. the solutions all converge to $\bar{p}$. (See $[3, \S 4.3]$.

Stability of $\bar{p}$ in $X_{+}$can be seen as follows. Suppose that $\left|p_{0}-\bar{p}\right|_{X} \leq \delta \leq 1$. Then the Lyapunov functional $\Phi_{0}$ implies

$$
|y(t)-\bar{y}|_{2}+|u(t)|_{1}+|z(t)|_{1}+|w(t)|_{2} \leq C \delta \quad t>0
$$


with some constant $C$ which only depends on $G$ and on $\xi, \alpha^{\prime}$ and $R_{0}$. Since the solutions are uniformly bounded we may conclude

$$
|p(t)-\bar{p}|_{p} \leq C_{1} \delta^{1 / p}, \quad t>0
$$

with some uniform constant $C_{1}$ and $p \in(1, \infty)$. Now we use the equation for $u$ and Sobolev embedding (choose $p>n$ ) to obtain

$$
|u(t)|_{\infty} \leq\left|e^{-\delta_{u} D_{N} t-\xi t} u_{0}\right|_{\infty}+c\left|e^{-\delta_{u} D_{N} t-\xi t} * z\right|_{W_{p}^{1}(G)} \leq \delta+c \sup _{t>0}|z(t)|_{p} \leq c \delta^{1 / p} .
$$

This way we see that $u(t)$ stays small in $C(\bar{G})$. The same argument can be applied to show smallnes of $|w(t)|_{\infty}$. Next we write

$$
\partial_{t}(y-\bar{y})=-\rho(y-\bar{y})-u y
$$

hence

$$
|y(t)-\bar{y}|_{\infty} \leq e^{-\rho t}\left|y_{0}-\bar{y}\right|_{\infty}+M \int_{0}^{t} e^{-\rho(t-s)}|u(s)|_{\infty} d s \leq \delta+M \sup _{t>0}|u(t)|_{\infty} / \rho,
$$

where $M=\sup _{t>0}|y(t)|_{\infty}$. This estimate shows that $|y(t)-\bar{y}|_{\infty}$ stays small as well. For $z$ we can use the same argument. In conclusion, for given $\varepsilon>0$ we find $\delta>0$ such that $|p(t)-\bar{p}|_{X} \leq \varepsilon$ for $t>0$ whenever $p_{0} \in X_{+},\left|p_{0}-\bar{p}\right|_{X} \leq \delta$. Thus $\bar{p}$ is stable in $X_{+}$.

(vi) The Case $R_{0}>1$

Now assume $R_{0}>1$. This time we restrict attention to $D:=\operatorname{int} X_{+}$which is positive invariant by (iv). The Lyapunov functional is in this case

$$
\Phi(p)=\int_{G}\left[\frac{\tilde{y}}{\xi} \phi(u, \tilde{u})+\phi(y, \tilde{y})+\phi(z, \tilde{z})+\frac{1}{2 \alpha^{\prime}}(w-\tilde{w})^{2}\right] d x,
$$

where $\phi(u, \tilde{u})=u-\tilde{u}-\tilde{u} \log (u / \tilde{u})$. Note that $\phi(\cdot, \tilde{u})$ is convex and has the strict minimum 0 at $u=\tilde{u}$, and we have the lower bound

$$
\phi(u, \tilde{u}) \geq \frac{\tilde{u}}{2 u_{\infty}^{2}}(u-\tilde{u})^{2}, \quad 0<u<u_{\infty}
$$

$\Phi$ is well-defined and of class $C^{1}$ on $D$, and the derivative along solutions becomes

$$
\begin{aligned}
\partial_{t} \Phi(p(t)) & =-\frac{\tilde{y} \delta_{u} \tilde{u}}{\xi} \int_{G} \frac{|\nabla u|^{2}}{u^{2}} d x-\frac{\delta_{w}}{\alpha^{\prime}} \int_{G}|\nabla w|^{2} d x-\frac{\beta}{\alpha^{\prime}} \int_{G}(w-\tilde{w})^{2} d x \\
& -\int_{G} \frac{\rho}{y}(y-\tilde{y})^{2} d x-\tilde{u} \tilde{y} \int_{G}\left[\frac{z}{\xi u}+\frac{\tilde{y}}{y}+\frac{\xi u y}{\tilde{y} z}-3\right] d x \leq 0,
\end{aligned}
$$

cp. the proof of Theorem 2..2. Hence $\Phi$ is a Lyapunov functional on $D$, and it is even strict. Next we show that for an initial value $p_{0} \in D$ we have $\omega\left(p_{0}\right) \subset D$. In fact, let $p_{\infty} \in \omega\left(p_{0}\right)$; choose a sequence $t_{n} \rightarrow \infty$ such that $p\left(t_{n}\right) \rightarrow p_{\infty}$. Fatou's lemma yields

$$
\Phi\left(p_{\infty}\right) \leq \liminf _{n \rightarrow \infty} \Phi\left(p\left(t_{n}\right)\right) \leq \Phi\left(p_{0}\right)<\infty,
$$


and this implies $u_{\infty}>0$ a.e. But then we must have $p_{\infty} \in D$ since $p_{\infty}$ lies on a complete orbit. Since $\Phi$ is a strict Lyapunov functional on $D$, we have $\omega\left(p_{0}\right)=\{\tilde{p}\}$, i.e. $\tilde{p}$ is globally attractive in $D$. Since all solutions with $p_{0} \in X_{+}$and $u_{0}+z_{0} \neq 0$ immediately enter $D$ we see that $\tilde{p}$ attracts all solutions with such initial values.

Stability of $\tilde{p}$ is obtained as in (v) since $\Phi(p) \geq c|p-\tilde{p}|_{2}^{2}$ with some constant $c>0$ depending only on the parameters and $\left|p_{0}\right|_{X}$.

Finally, instability of $\bar{p}$ follows from the attractivity properties of $\tilde{p}$.

The proof of Theorem 3.1 essentially follows the same lines except for some minor differences which we are going to comment.

(i) The subsystem $T \equiv 0$, i.e. the model without immune response is treated exactly as in the proof of Theorem 3.2 which yields assertion (d) of Theorem 3.1. Therefore we may assume $w_{0} \in C_{+}(\bar{G})$, $w_{0} \neq 0$.

(ii) The first modification concerns the uniform bounds. We obtain uniform bounds on $u, y$ and $z$ as in the proof of Theorem 3.2, however, due to the nonlinear term $\alpha w z$ in the equation for $w$ we have to use a different argument for $w$. Integrating the equations for $y, z$ and $w$ w.r.t. $x$ we obtain

$$
\partial_{t} \int_{G}[y+z+w / \alpha] d x=\int_{G}[\sigma-\rho y-z-\beta w / \alpha] d x \leq \sigma|G|-\rho_{0} \int_{G}[y+z+w / \alpha] d x,
$$

with $\rho_{0}:=\min \{\rho, 1, \beta\}>0$. This implies in particular $|w(t)|_{1} \leq M$ for $t>0$, with some constant $M>0$. This and the uniform bound on $z$ yield $z w \in L_{\infty}\left(\mathbb{R}_{+} ; L_{1}(G)\right)$. Denote by $D_{N}^{1}$ the Neumann Laplacian on $L_{1}(G)$. Then the real interpolation space $\left(L_{1}(G), D\left(D_{N}^{1}\right)\right)_{\theta, 1}$ is embedded into $\left.W_{1}^{2 \theta}(G)\right)$ for $\theta \in(1 / 2,1)$. This fact is shown in [1] for the Dirichlet Laplacian, and the proof given there extends to our case in view of the estimates for the corresponding semigroup given in [10, Thm.5.7]. The equation for $w$ then implies $w \in L_{\infty}\left(\mathbb{R}_{+} ; W_{1}^{2-\varepsilon}(G)\right)$ by $[5, \S 2.2 .1]$, for $\varepsilon \in(0,2)$. Hence the Sobolev embedding

$$
w \in L_{\infty}\left(\mathbb{R}_{+} ; W_{1}^{2-\varepsilon}(G)\right) \hookrightarrow L_{\infty}\left(\mathbb{R}_{+} ; L_{p_{1}}(G)\right)
$$

with $\frac{1}{p_{1}}=1-\frac{2-\varepsilon}{n}$ yields $z w \in L_{\infty}\left(\mathbb{R}_{+} ; L_{p_{1}}(G)\right)$, so that $w \in L_{\infty}\left(\mathbb{R}_{+} ; W_{p_{1}}^{2-\varepsilon}(G)\right)$. (We may assume that $n \geq 2$.) Sobolev's embedding then implies $w \in L_{\infty}\left(\mathbb{R}_{+} ; L_{p_{2}}(G)\right)$, with $\frac{1}{p_{2}}=\frac{1}{p_{1}}-\frac{2-\varepsilon}{n}$. Iterating this argument, after finitely many, say $k$, steps we have $p_{k}>n$ and then after one more iteration step we may conclude $w \in L_{\infty}\left(\mathbb{R}_{+} ; C(\bar{G})\right)$.

(iii) For strict positivity we have to assume here $p_{0} \in X_{+}$and $w_{0} \neq 0, u_{0}+z_{0} \neq 0$. Employing the Lyapunov functional

$$
\Phi(p)=\int_{G}\left[\frac{\hat{y}}{\xi} \phi(u, \hat{u})+\phi(y, \hat{y})+\phi(z, \hat{z})+\frac{1}{\alpha} \phi(w, \hat{w})\right] d x
$$

we obtain assertion (c) as in (vi) of the proof of Theorem 3.2. 
(iv) For the proof of assertion (b) we use the Lyapunov functional

$$
\Phi_{*}(p)=\int_{G}\left[\phi\left(u, u_{*}\right)+\phi\left(y, y_{*}\right)+\phi\left(z, z_{*}\right)+\frac{w}{\alpha}\right] d x
$$

and for assertion (a)

$$
\Phi_{0}(p)=\int_{G}\left[\frac{1}{2}(y-\bar{y})^{2}+(2 \xi-\bar{y})\left(u+z+\frac{1}{\alpha} w\right] d x\right.
$$

One easily computes that these are strict Lyapunov functionals for (5..1) with $i=1$, and then we may again argue as in the proof of Theorem 3.2.

\section{References}

[1] G. Di Blasio. Analytic semigroups generated by elliptic operators in $L^{1}$ and parabolic equations. Osaka J. Math. 28 (1991), 367-384.

[2] P. Georgescu, Ying-Hen Hsieh. Global stability for a virus dynamics model with nonlinear incidence of infection and removal. SIAM J. Appl. Math. 67 (2006/07), 337-353.

[3] D. Henry. Geometric theory of semilinear parabolic equations. Springer-Verlag, Berlin, New York, 1981.

[4] A. Korobeinikov. Global properties of basic virus dynamics models. Bull. Math. Biology, 66 (2004), 879-883.

[5] A. Lunardi. Analytic Semigroups and optimal regularity in parabolic problems. Birkhäuser Verlag, Basel, 1995.

[6] M.A Nowak, R.M May. Virus dynamics. Oxford University Press, Oxford, 2000.

[7] A. Pazy. Semigroups of linear operators and applications to partial differential equations. Springer, Berlin 1983.

[8] M.H. Protter, H.F. Weinberger. Maximum principles in differential equations. Prentice-Hall, Englewood Cliffs (NJ), 1967.

[9] J. Prüss, L. Pujo-Menjouet, G.F. Webb. R. Zacher. Analysis of a model for the dynamics of prions. Discrete Contin. Dyn. Syst. Ser. B 6 (2006), 225-235 .

[10] H. Tanabe. Functional analytic methods for partial differential equations. Marcel Dekker, New York, 1997.

[11] D. Wodarz. Killer cells dynamics. Mathematical and computational approaches to immunology. Springer-Verlag, New York, 2006. 